\title{
Chymotrypsin- and trypsin-like activities secreted by the multidrug- resistant yeasts forming the Candida haemulonii complex
}

\author{
XÊNIA M. SOUTO ${ }^{1}$, MARTA H. BRANQUINHA ${ }^{1}$ and ANDRÉ L.S. SANTOS ${ }^{1,2}$ \\ ${ }^{1}$ Universidade Federal do Rio de Janeiro/UFRJ, Instituto de Microbiologia Paulo de Góes, Centro de Ciências \\ da Saúde, Departamento de Microbiologia Geral, Laboratório de Estudos Avançados de Microrganismos \\ Emergentes e Resistentes/LEAMER, Av. Carlos Chagas Filho, 373, 21941-901 Rio de Janeiro, RJ, Brazil \\ ${ }^{2}$ Programa de Pós-Graduação em Bioquímica, Instituto de Química, Universidade Federal do Rio de \\ Janeiro/UFRJ, Av. Athos da Silveira Ramos, 149, 21941-909 Rio de Janeiro, RJ, Brazil
}

Manuscript received on July 23, 2018; accepted for publication on October 18, 2018

\begin{abstract}
How to cite: SOUTO XM, BRANQUINHA MH AND SANTOS ALS. 2019. Chymotrypsin- and trypsin-like activities secreted by the multidrug-resistant yeasts forming the Candida haemulonii complex. An Acad Bras Cienc 91: e20180735. DOI 10.1590/0001-3765201920180735.
\end{abstract}

\begin{abstract}
Candida haemulonii complex (C. haemulonii, $C$. haemulonii var. vulnera and $C$. duobushaemulonii) consists of emergent multidrug-resistant pathogens that cause bloodstream and deepseated infections. However, little is known about their virulence factors. Herein, we evaluated the presence of extracellular serine peptidases in this fungal complex. Serine peptidase activity was measured by spectrophotometry using chromogenic peptide substrates to the S1 family. Chymotrypsin-, trypsin- and elastase-like activities were detected in all fungal isolates. Since higher chymotrypsin- and trypsin-like activities were observed from the cleavage of $N$-succinyl-Ala-Ala-Pro-Phe- $p$ Na and $N$-benzoyl-Phe-ValArg- $p \mathrm{Na}$, respectively, these substrates were selected for further experiments. Overall, pHs 7.0 and 9.0 were those in which higher chymotrypsin- and trypsin-like activities were observed, respectively, displaying higher hydrolytic activities at $37-45^{\circ} \mathrm{C}$. Additionally, the serine peptidases produced by C. haemulonii complex were inhibited by PMSF and AEBSF in a typically concentration-dependent manner. Although the Michaelis constant $\left(\mathrm{K}_{\mathrm{m}}\right)$ values obtained for chymotrypsin-like peptidases were similar, greater differences were observed for trypsin-like enzymes secreted by the different fungal isolates. This is the first time that peptidases belonging to the $\mathrm{S} 1$ family are described in the $C$. haemulonii species complex. Thus, these data open the doors for more detailed studies into potential roles of these peptidases in fungal virulence.
\end{abstract}

Key words: Brazilian clinical isolates, Candida haemulonii complex, chymotrypsin, serine peptidases, trypsin, virulence factors.

\section{INTRODUCTION}

Candidiasis is one of the most frequent nosocomial infections worldwide and has drawn attention, in recent years, due to its significant contribution to the

Correspondence to: André Luis Souza dos Santos

E-mail: andre@micro.ufrj.br

ORCid: https://orcid.org/0000-0003-0821-8592 increased mortality rate of immunocompromised/ immunosuppressed patients (Lockhart 2014, Agarwal et al. 2017). Although Candida albicans remains the most commonly isolated fungal species in hospital settings, infections by non-albicans Candida species have been increasingly reported over the last decades in a global scale (Agarwal et al. 2017). 
The Candida haemulonii complex is composed by three phenotypically indistinguishable fungal species: C. haemulonii, C. duobushaemulonii and $C$. haemulonii var. vulnera. Relevantly, these emerging yeasts are intrinsically resistant to the main antifungals commonly used in the clinical arena, including amphotericin B and different azoles (e.g., fluconazole, itraconazole and voriconazole) as well as they present limited susceptibility to echinocandins. Consequently, this multidrugresistant profile has hampered the treatment of patients with systemic infections and increased the number of clinical failures and related cases of death caused by these fungal species (Rodero et al. 2002, Khan et al. 2007, Kim et al. 2009, Ruan et al. 2010, Crouzet et al. 2011, Cendejas-Bueno et al. 2012, Li et al. 2015, Ramos et al. 2015, Fang et al. 2016). Although C. haemulonii species complex infections have gained prominence in recent years, the interactions of these yeasts with the host remain poorly studied. In order to change this scenario, our research group has been engaged since 2015 in characterizing several aspects of the physiology and virulence of these emerging pathogens, such as antifungal susceptibility, biofilm formation and secretion of hydrolytic enzymes (Ramos et al. 2015, 2016, 2017). In this context, Ramos et al. (2016) described the production of aspartic-type peptidase in 12 Brazilian clinical isolates of $C$. haemulonii species complex, as judged by the degradation of albumin molecules incorporated into agar plates, in which $C$. haemulonii and $C$. haemulonii var. vulnera were classified as good producers and C. duobushaemulonii as excellent producers. In addition, caseinolytic activity was detected in all tested isolates, suggesting the secretion of other peptidase classes by this fungal complex.

Historically, several papers have pointed out to the relevance of secreted serine peptidases as virulence factors in fungi (Muszewska et al. 2017). In this sense, serine peptidases are involved in different contexts of fungal-host interplays, and they are important for nutrient acquisition from proteinrich sources (Reddy et al. 1996, Muszewska et al. 2017). In Candida spp., serine peptidases presenting hydrolytic activities in a wide range of $\mathrm{pH}$ as well as with the ability to hydrolyze key host proteins, such as components of the extracellular matrix and serum proteins, have already been described in $C$. albicans, C. guilliermondii, C. parapsilosis, C. tropicalis, C. dubliniensis and C. lipolytica (Santos and Soares 2005, Santos et al. 2006, Melo et al. 2007, Vermelho et al. 2010, Portela et al. 2010). Secreted serine-type peptidases belonging to the $\mathrm{S} 1$ family (e.g., trypsin-, chymotrypsin- and elastaselike peptidases) (Dubovenko et al. 2010) have gained prominence due to their multiple roles in the pathogenicity of opportunistic fungi (Muszewska et al. 2017), such as Scedosporium, Sporothrix and Aspergillus (Muhsin et al. 1997, Barata et al. 2002, Han et al. 2017). These serine peptidases have great similarities in their sequences and tridimensional structures; however, they present different substrate specificities (Haën et al. 1975, Ma et al. 2005), in which trypsin-like peptidases have affinity for basic amino acid residues (e.g., lysine and arginine), chymotrypsin-like peptidases have affinity for aromatic amino acid residues (e.g., phenylalanine, tyrosine and tryptophan) (Vajda and Szabó 1976), while elastase-like peptidases have preference for aliphatic amino acid residues, particularly alanine (Zimmerman and Ashe 1977).

So far, almost nothing is known about the secretion of serine-type peptidases by $C$. haemulonii species complex (Ramos et al. 2016, Souto et al. 2019). In this context and bearing in mind the importance of the S1 family of serine peptidases in many aspects of the fungus-host interaction, in the present study we have described the presence of serine peptidases in clinical isolates of $C$. haemulonii, C. haemulonii var. vulnera and C. duosbushaemulonii. 


\section{MATERIALS AND METHODS}

\section{CHEMICALS}

The chromogenic peptide substrates $N$-succinylAla-Ala-Pro-Phe- $p \mathrm{Na}$ (chymotrypsin detection), $N$-succinyl-Ala-Ala-Pro-Leu- $p \mathrm{Na}, N$-succinylAla-Ala-Ala- $p \mathrm{Na}$ (elastase detection), $N$-benzoyl-Phe-Val-Arg- $p \mathrm{Na}$ and $N \alpha$-benzoylDL-4Arg- $p \mathrm{Na}$ (trypsin detection), the peptidase inhibitors phenylmethanesulfonyl fluoride (PMSF), 4-(2-aminoethyl)benzenesulfonyl fluoride hydrochloride (AEBSF), $N$ - $p$-tosyl-L-

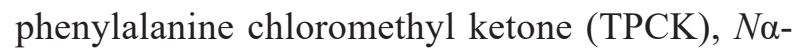
tosyl-L-lysine chloromethyl ketone hydrochloride (TLCK), benzamidine, 1,10-phenanthroline, trans-epoxysuccinyl-L-leucylamido(4-guanidino) butane (E-64) and pepstatin A, as well as bovine serum albumin (BSA) were obtained from SigmaAldrich (St Louis, MO, USA). Buffer components were purchased from Isofar (Duque de Caxias, RJ, Brazil). Culture medium components were provided by Neogen (Lansing, MI, USA). All other reagents were of analytical grade.

\section{MICROORGANISMS AND GROWTH CONDITIONS}

Three Brazilian clinical isolates of the C. haemulonii complex were used in all the experiments performed in the present study: C. haemulonii (LIPCh7 from toenail), C. haemulonii var. vulnera (LIPCh9 from urine) and C. duobushaemulonii (LIPCh10 from bronchoalveolar lavage) (Ramos et al. 2015). Fungi were cultured in Sabouraud-dextrose medium (400 $\mathrm{ml})$ at $37^{\circ} \mathrm{C}$ for $48 \mathrm{~h}$ under constant agitation (200 $\mathrm{rpm})$. The clinical fungal isolates studied herein have reached their exponential phase at $48 \mathrm{~h}$ of in vitro growth on Sabouraud-dextrose liquid medium as previously published by our research group (Ramos et al. 2017).

\section{COLLECTION OF THE CULTURE SUPERNATANTS}

The fungal cultures were harvested by centrifugation $\left(4.000 \times \mathrm{g}, 10 \mathrm{~min}, 4^{\circ} \mathrm{C}\right)$ and the supernatants were filtered in a $0.22-\mu \mathrm{m}$ membrane (Millipore, São Paulo, Brazil). The cell-free culture supernatants were concentrated 100-fold in an Amicon ${ }^{\circledR}$ ultrafiltration system (Amicon ${ }^{\circledR}$, Beverly, USA) using a 10-kDa cut-off membrane (Santos and Soares 2005). The same volume of Sabouraud medium was also concentrated and used as a control to check for possible peptidase activity, showing negative results. Protein concentration of the culture supernatants was determined by the method described by Lowry et al. (1951), using BSA as standard.

\section{CHARACTERIZATION OF EXTRACELLULAR SERINE PEPTIDASE ACTIVITY}

The extracellular serine peptidase activities were determined, initially, in $10 \mathrm{mM}$ sodium phosphate buffer ( $\mathrm{pH}$ 7.0) using $p$ NA-labeled peptide substrates for chymotrypsin, elastase and trypsin. Then, substrates displaying the highest hydrolysis for serine peptidase activities were selected for further studies as described below.

Modulation of serine peptidase activity by different $\mathrm{pHs}$ and temperatures was evaluated, respectively, using $0.1 \mathrm{M}$ sodium citrate ( $\mathrm{pH}$ 5.0), $0.1 \mathrm{M}$ citrate phosphate ( $\mathrm{pH} 6.0), 10 \mathrm{mM}$ sodium phosphate ( $\mathrm{pH} 7.0), 0.1 \mathrm{M}$ Tris- $\mathrm{HCl}(\mathrm{pH} 8.0), 0.1$ $\mathrm{M}$ sodium glycine-hydroxide ( $\mathrm{pH} 9.0-10.0)$ and $50 \mathrm{mM}$ sodium phosphate $(\mathrm{pH} 11.0)$ as buffer solutions, and by incubating the supernatants at $25,37,45$ or $55^{\circ} \mathrm{C}$. For this last analysis, the $\mathrm{pH}$ of higher activity for each selected substrate was used. In addition, all other assays were performed at $37^{\circ} \mathrm{C}$. The effects of proteolytic inhibitors on peptidase activities were evaluated after incubation with PMSF (0.0001-1 mM), AEBSF (0.1-10 mM), benzamidine (1-10 mM), 1,10-phenanthroline (1$10 \mathrm{mM})$, TPCK (0.01-0.2 mM), TLCK (0.01-0.2 $\mathrm{mM})$, E-64 $(0.01 \mathrm{mM})$ or pepstatin A $(0.01 \mathrm{mM})$. Systems with no proteolytic inhibitor were used as controls. The enzymatic activities assayed in the absence of inhibitors was considered as 
$100 \%$. Kinetic parameters were determined by incubating the culture supernatants with increasing concentrations of the substrates for which higher serine peptidase activities were detected. The apparent $\mathrm{K}_{\mathrm{m}}$ and $\mathrm{V}_{\text {max }}$ for these substrates were determined by non-linear regression analysis applying Michaelis-Menten kinetics using the Prism v5.03 program (GraphPad Software, Inc.). For all assays, supernatants to a final concentration of $0.5 \mu \mathrm{g} / \mu 1$ total extracellular proteins were added to different substrates (final concentration ranging from 0.1 to $1 \mathrm{mM}$ for kinetic parameters, and $100 \mu \mathrm{M}$ for further analysis) in the desired buffer solutions. The release of $p \mathrm{Na}$ was evaluated in 96well plates by spectrophotometry at $410 \mathrm{~nm}$ during 60 min of incubation at 5-min intervals between each reading. The specific serine peptidase activity was expressed as nmol of $p \mathrm{Na}$ released per min and $\mathrm{mg}$ of total protein in concentrated culture supernatants. The concentration of $p \mathrm{Na}$ was calculated using a molecular extinction coefficient of $8270 \mathrm{M}^{-1} \cdot \mathrm{cm}^{-1}$. The effect of inhibitors was expressed as percentage of residual peptidase activity in relation to untreated controls.

\section{STATISTICAL ANALYSIS}

All the experiments were performed in triplicate, in three independent experimental sets. Data were expressed as mean \pm standard deviation. The results were evaluated by analysis of variance (ANOVA) followed by Tukey's test using the Prism v5.03 program (GraphPad Software, Inc.). In all analyses, $P$ values $\leq 0.05$ were considered statistically significant.

\section{RESULTS}

SECRETION OF SERINE PEPTIDASES BELONGING TO THE S1 FAMILY

Initially, secretion of serine peptidases belonging to the S1 family was evaluated in the culture supernatants from three clinical isolates of the
C. haemulonii complex employing five distinct substrates to detect chymotrypsin-, trypsin- and elastase-like activities. These three types of enzymatic classes were detected in all supernatants (Fig. 1). Interestingly, no activity was detected against the $N \alpha$-benzoyl-DL-4Arg- $p$ Na substrate, typical for trypsin. With respect to the other substrates, higher serine peptidase activities were detected, after $60 \mathrm{~min}$, in the supernatant of $C$. haemulonii followed by C. duobushaemulonii and C. haemulonii var. vulnera (Fig. 1, insert).

\section{BIOCHEMICAL PROPERTIES OF CHYMOTRYPSIN-} AND TRYPSIN-LIKE PEPTIDASES

Since higher activities were detected over $N$-succinyl-Ala-Pro-Phe- $p \mathrm{Na}$ and $N$-benzoylPhe-Val-Arg- $p \mathrm{Na}$, these substrates were selected to investigate the biochemical properties of chymotrypsin- and trypsin-like peptidases secreted by $C$. haemulonii species complex, respectively.

\section{EFFECT OF pH AND TEMPERATURE}

In general, serine peptidase activities were detected in a wide $\mathrm{pH}$ range (varying from 5.0 to 11.0 ) (Fig. 2). However, for all analyzed samples, pHs 7.0 and 9.0 were those of greater chymotrypsinand trypsin-like activities, respectively. Besides that, these hydrolytic activities were higher at temperatures of $37-45^{\circ} \mathrm{C}$, although activities were also detected at 25 and $55^{\circ} \mathrm{C}$ (Fig. 3).

\section{EFFECT OF PEPTIDASE INHIBITORS}

The effect of inhibitors against serine peptidase activities are shown in Table I. Chymotrypsin-like activities detected in supernatants of all fungal isolates were inhibited by AEBSF and PMSF, in a concentration-dependent manner. At higher concentrations, AEBSF (10 mM) and PMSF (0.1 $\mathrm{mM}$ ) reduced the enzymatic activities up to 80 and $100 \%$, respectively. Besides that, TPCK $(0.2 \mathrm{mM})$ also induced a slight reduction $(\approx 18 \%)$ on these 


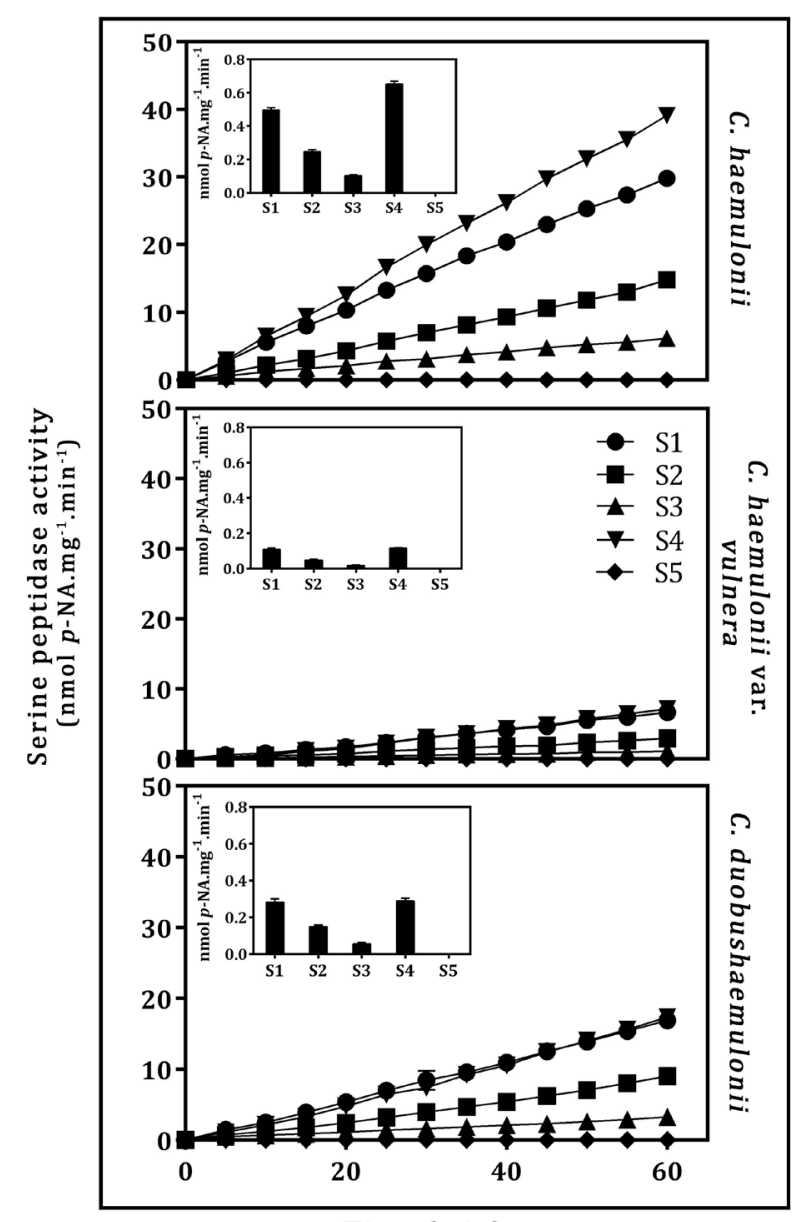

Time (min)

Figure 1 - Serine peptidase activities measured in the culture supernatants of clinical isolates of the $C$. haemulonii complex. The serine peptidase activities were evaluated during $60 \mathrm{~min}$ of incubation at $\mathrm{pH} 7.0$ and $37^{\circ} \mathrm{C}$. Five substrates were used: $N$-succinyl-Ala-Ala-Pro-Phe- $p \mathrm{Na}(\mathrm{S} 1)$ for chymotrypsin, $N$-succinyl Ala-Ala-Pro-Leu- $p \mathrm{Na}$ (S2) and $N$-succinyl-AlaAla-Ala- $p \mathrm{Na}$ (S3) for elastase, and $N$-benzoyl-Phe-Val-Arg$p \mathrm{Na}$ (S4) and $N \alpha$-benzoyl-DL-4Arg- $p \mathrm{Na}$ (S5) for trypsin. The inserts show the average of serine peptidase activity against each substrate at $60 \mathrm{~min}$. Values represent means $\pm \mathrm{SD}$ from at least three independent experiments.

peptidase activities. In a similar way, trypsin-like activities detected in all evaluated supernatants were also inhibited by AEBSF and PMSF in a typically concentration-dependent fashion. Additionally, the inhibition of these trypsin-like activities by approximately 100,75 and $40 \%$ was measured in the presence of $10 \mathrm{mM}$ AEBSF, 1 mM PMSF and $10 \mathrm{mM}$ benzamidine, respectively.
Differently, TLCK, 1,10-phenanthroline, E-64 and pepstatin A did not affect the hydrolytic activities of both chymotrypsin- and trypsin-like peptidases.

\section{KINETIC PARAMETERS}

The kinetic parameters of the chymotrypsinand trypsin-like peptidases detected in the $C$. haemulonii, C. hameulonii var. vulnera and $C$. duobushaemulonii secretions were also evaluated (Table II). In general, apparent $\mathrm{K}_{\mathrm{m}}$ and $\mathrm{V}_{\max }$ values for $N$-succinyl-Ala-Ala-Pro-Phe- $p \mathrm{Na}$ ranged from 0.96 to $0.99 \mathrm{mM}$ and 1.09 to $7.0 \mathrm{nmol} p \mathrm{NA}_{\mathrm{mg}}{ }^{-1}$. $\mathrm{min}^{-1}$, respectively. In turn, for $\mathrm{N}$-benzoyl-PheVal-Arg- $p \mathrm{Na}$, apparent $\mathrm{K}_{\mathrm{m}}$ ranged from 0.098 to $0.17 \mathrm{mM}$ and $\mathrm{V}_{\max }$ from 1.0 to $1.87 \mathrm{nmol} p \mathrm{NA}$. $\mathrm{mg}^{-1} \cdot \mathrm{min}^{-1}$. By one-way ANOVA test, no significant difference was observed between the apparent $\mathrm{K}_{\mathrm{m}}$ values found for the chymotrypsin-like serine peptidases. On the other hand, the apparent $\mathrm{K}_{\mathrm{m}}$ value found for the trypsin-like serine peptidase produced by $C$. haemulonii differed significantly $(P<0.05)$ from those found in $C$. haemulonii var. vulnera and C. duobushaemulonii.

\section{DISCUSSION}

In recent years, the rapid emergence of multidrug resistant non-albicans Candida species, such as those belonging to the C. haemulonii complex, has stimulated the search for new therapeutic agents and specifically serine peptidase inhibitors have emerged as a valuable alternative to test their antifungal potential (Santos and Soares 2005, Santos et al. 2006, Melo et al. 2007, Vermelho et al. 2010, Portela et al. 2010, Santos 2011, Whaley et al. 2016). Among the secreted serine peptidases correlated with the fungal pathogenesis in humans, we can highlight those belonging to the S1 family (one of the main families of fungal serine peptidases), which are well described in several genera of filamentous fungi, but very little studied in Candida spp. (Muhsin et al. 1997, Barata et al. 2002, Zarnowski et al. 2007, Dubovenko et al. 2010, Muszewska 


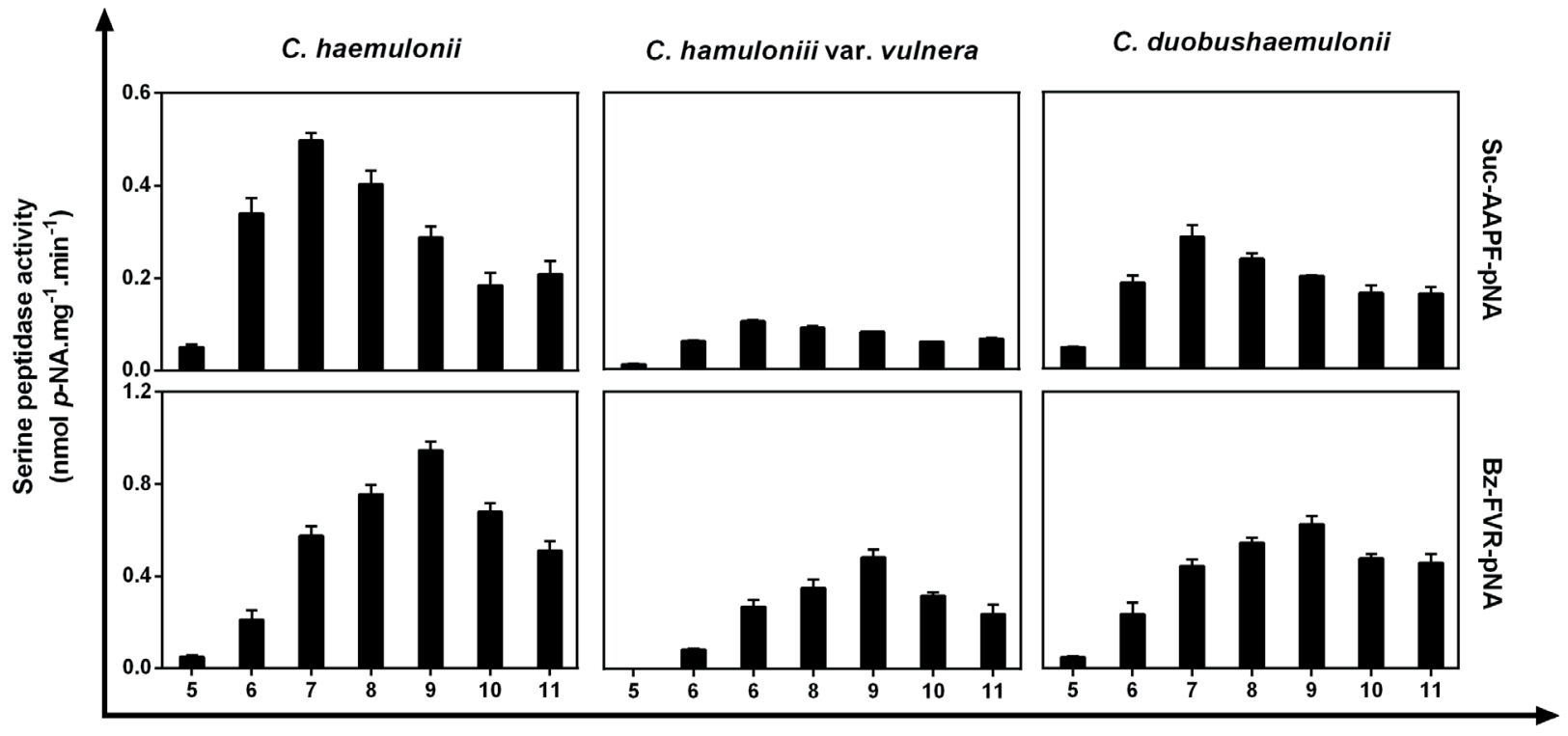

$\mathrm{pH}$

Figure 2 - Effect of $\mathrm{pH}$ on chymotrypsin- and trypsin-like activities measured in the culture supernatants of clinical isolates of the C. haemulonii complex. The serine peptidase activities were evaluated by spectrophotometry in a $\mathrm{pH}$ range varying from 5.0 to 11.0 for $60 \mathrm{~min}$ at $37^{\circ} \mathrm{C}$. Two substrates were used: $N$-succinyl-Ala-Ala-Pro-Phe- $p$ Na for chymotrypsin and $N$-benzoyl-Phe-ValArg- $p \mathrm{Na}$ for trypsin. The bars represent the mean $\pm \mathrm{SD}$ from at least three independent experiments.

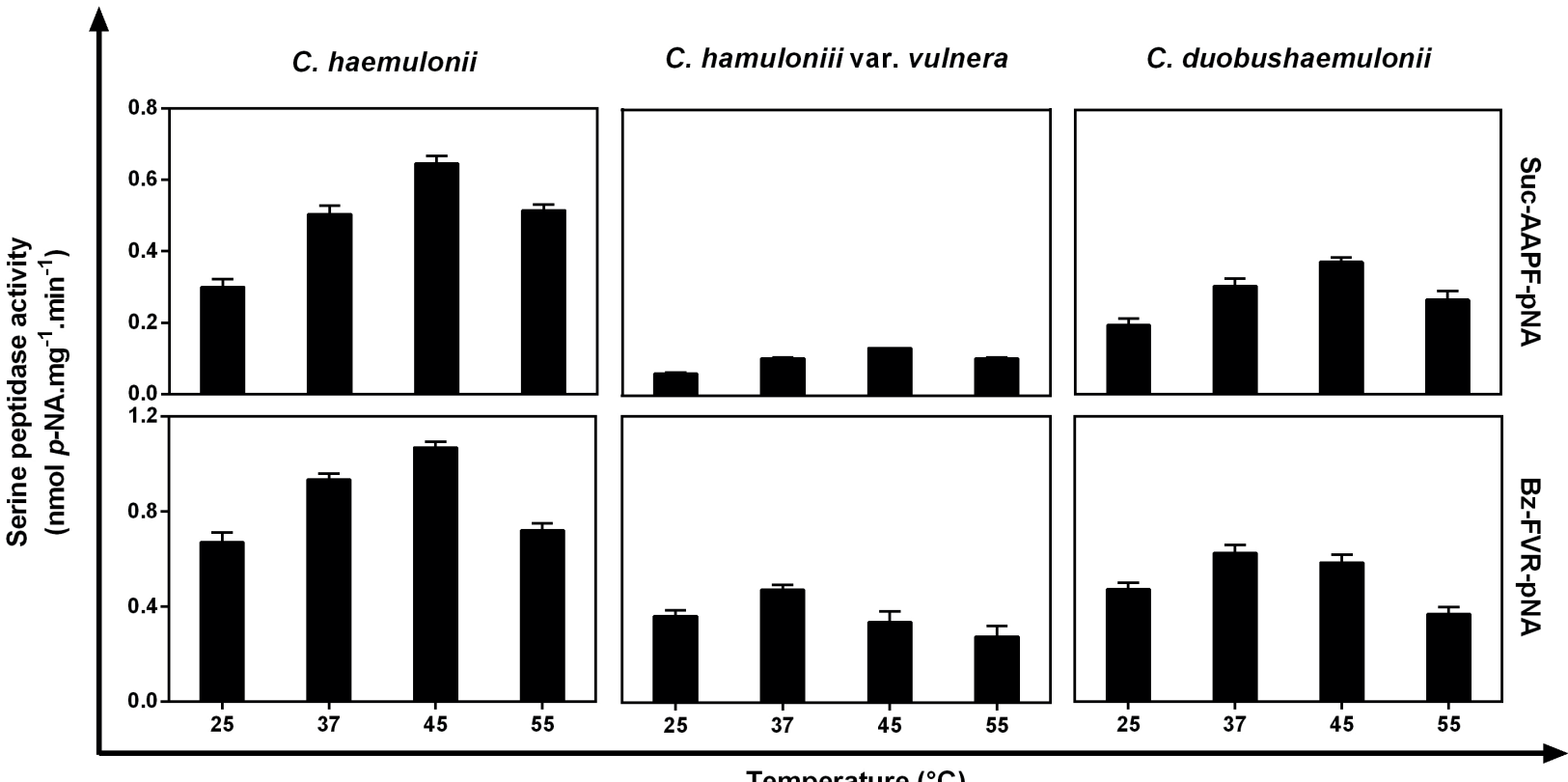

Figure 3 - Effect of temperature on chymotrypsin- and trypsin-like activities measured in the culture supernatants of clinical isolates of the $C$. haemulonii complex. The serine peptidase activities were evaluated by spectrophotometry for 60 min at 25,37 , 45 and $55^{\circ} \mathrm{C}$. Two substrates were used: $N$-succinyl-Ala-Ala-Pro-Phe- $p$ Na for chymotrypsin and $N$-benzoyl-Phe-Val-Arg- $p$ Na for trypsin, at $\mathrm{pH}$ 9.0. The bars represent the mean $\pm \mathrm{SD}$ from at least three independent experiments. 
et al. 2017). In the literature, several studies have proposed that serine peptidases belonging to the $\mathrm{S} 1$ family play many pathophysiological roles, acting as virulence factors in different pathogenic fungi. In Sporothrix schenckii, for example, chymotrypsinlike serine peptidases enable the fungus to invade the skin and to obtain peptides from insoluble protein sources during the interaction with the host (Tsuboi et al. 1987). In the same way, secreted elastase-like serine peptidases from Aspergillus fumigatus assist this pathogen in lung infection during invasive arpergillosis (Kolattukudy et al. 1993). In turn, the secretion of trypsin-like peptidases has already been described in opportunistic pathogens such as Scedosporium aurantiacum (Han et al. 2017). Herein, the secretion of serine peptidases from the S1 family, including chymotrypsin-, trypsin- and elastase-like activities, was reported in C. haemulonii species complex.

Peptidases of microorganisms present unique characteristics with respect to the catalytic mechanism, substrate specificity, and optimal temperature and $\mathrm{pH}$ (Rao et al. 1998). Since peptidases participate in different aspects of the fungus-host interaction, their biochemical characterization is interesting not only for the understanding of the enzyme as a whole, but also for the knowledge of its possible functions during the infection with the objective of developing new therapeutic strategies. In view of this, secreted trypsin- and chymotrypsin-like serine peptidases, which provided the greatest activities in the clinical isolates used in the present study, were evaluated in relation to the influence of $\mathrm{pHs}$, temperatures and peptidase inhibitors on the enzyme activities, as well as regarding its kinetic parameters. Extracellular chymotrypsin- and trypsin-like serine peptidases exhibited activities in a wide range of pHs and temperatures. Since Candida spp. is exposed to a $\mathrm{pH}$ range from slightly alkaline to acidic (Davis 2009) and to possible temperature changes (Robert et al. 2015) during infection of the human host, the secretion of peptidases that adapt well to such variations probably will favor their survival, colonization and growth. In parallel, inhibition of both activities by PMSF and AEBSF confirmed the class of these enzymes as being serine peptidases. Besides that, the reduction of the hydrolysis of $N$-succinyl-Ala-Ala-Pro-Phe$p \mathrm{Na}$ and $N$-benzoyl-Phe-Val-Arg- $p \mathrm{Na}$ by TPCK and benzamidine, respectively, confirmed that these enzymes are chymotrypsin- and trypsin-like serine peptidases, since they are typical targets of such inhibitors. In agreement with these data, no inhibition was observed for pepstatin A, E-64 and 1,10-phenanthroline (inhibitors of aspartic-, cysteine- and metallopeptidases, respectively). Interestingly, TLCK, known to primarily inhibit trypsin-like serine peptidases, did not affect the activities of these enzymes. Secreted serine peptidases from the S1 family have been described mainly in human pathogenic, phytopathogenic and entomopathogenic fungi. In accordance with the results obtained here, a secreted chymotrypsinlike serine peptidase able to degrade the substrate $N$-succinyl-Ala-Ala-Pro-Phe- $p \mathrm{Na}$, with higher activity at $\mathrm{pH} 7.0$ and inhibited by PMSF, has been described in the entomopathogenic fungus Cordyceps sinensis (Zhang et al. 2008). A secreted trypsin-like serine peptidase able to degrade the substrate $N$-benzoyl-Phe-Val-Arg- $p \mathrm{Na}$, with higher activity at $\mathrm{pH} 8.5$ and inhibited by PMSF, was described in the phytopathogenic fungi Stagonospora nodorum (Carlile et al. 2000). With regard to human pathogenic fungi, secretion of chymotrypsin-like serine peptidases able to degrade the substrate $N$-succinyl-Ala-Ala-Pro-PheMCA and whose activity is inhibited by PMSF, was detected in the culture supernatant of the fungus $S$. schenckii (Tsuboi et al. 1987). In parallel, a secreted trypsin-like peptidase, with higher activity in basic $\mathrm{pH}$ at $45^{\circ} \mathrm{C}$ and inhibited by benzamidine, was described in the culture supernatant of Fusarium oxysporum (Barata et al. 2002). 


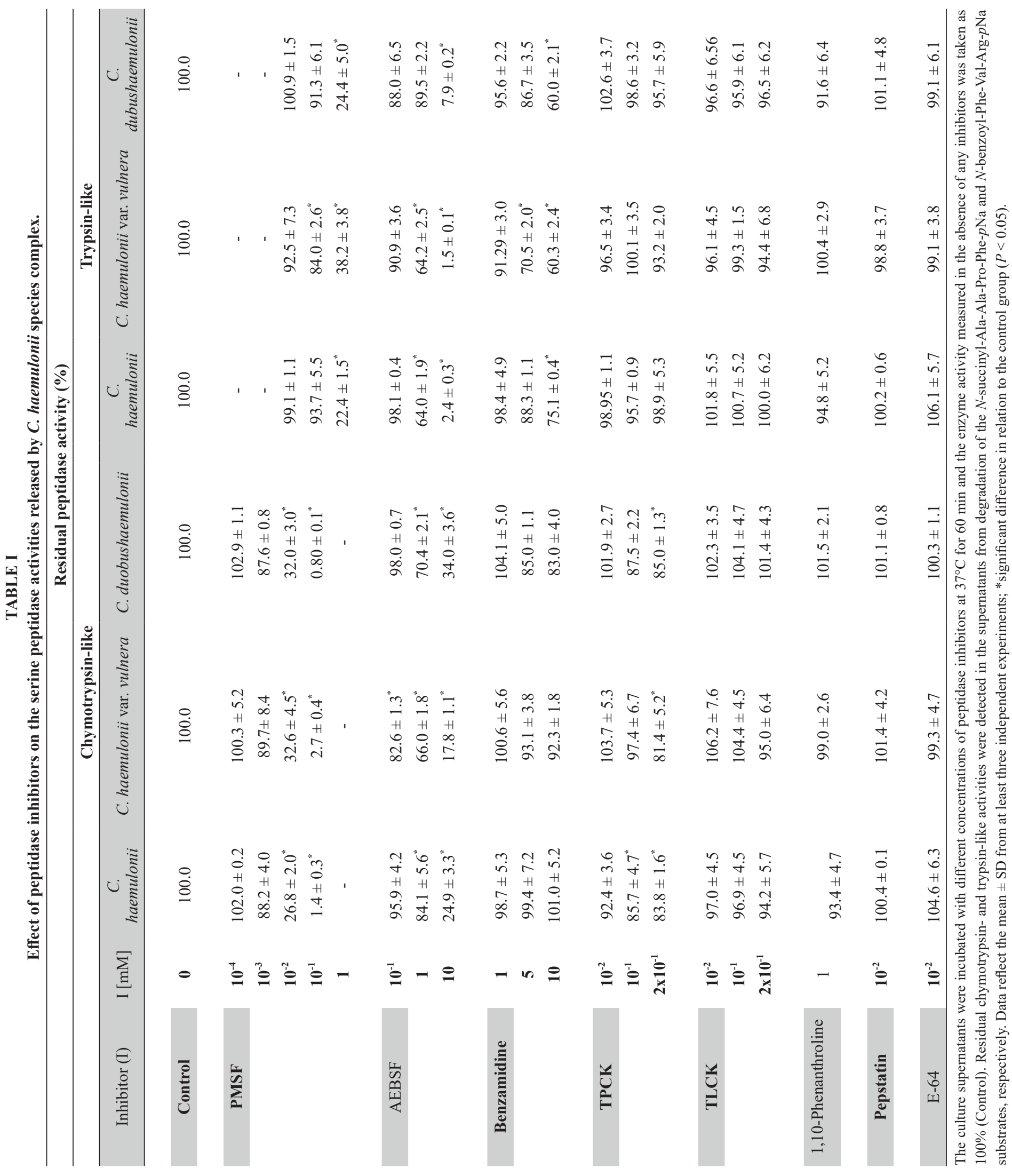


TABLE II

Kinetic parameters of the extracellular serine peptidase activities released by $C$. haemulonii species complex.

\begin{tabular}{|c|c|c|c|}
\hline Species & Serine-type peptidase & $K_{m}(m M)$ & $\mathrm{V}_{\max }\left(\mathrm{nmol} p \mathrm{NA} \cdot \mathrm{mg}^{-1} \cdot \mathrm{min}^{-1}\right)$ \\
\hline \multirow{2}{*}{ C. haemulonii } & Chymotrypsin-like & $0.96 \pm 0.12$ & $7.00 \pm 0.53$ \\
\hline & Trypsin-like & $0.10 \pm 0.01$ & $1.87 \pm 0.08$ \\
\hline \multirow{2}{*}{ C. haemulonii var. vulnera } & Chymotrypsin-like & $0.98 \pm 0.20$ & $1.09 \pm 0.14$ \\
\hline & Trypsin-like & $0.14 \pm 0.02$ & $1.00 \pm 0.04$ \\
\hline \multirow{2}{*}{ C. duobushaemulonii } & Chymotrypsin-like & $0.99 \pm 0.09$ & $4.52 \pm 0.27$ \\
\hline & Trypsin-like & $0.17 \pm 0.02$ & $1.71 \pm 0.08$ \\
\hline
\end{tabular}

In the present study, secreted serine peptidases from the S1 family were described, for the first time, in the C. haemulonii species complex, opportunistic fungal pathogens with extremely scarce knowledge about their pathophysiology processes. The broad range of $\mathrm{pH}$ and temperature at which these enzymes have hydrolytic activity suggests their abilities to function in several sites of human body and natural environments, presenting different physicochemical conditions. However, more accurate analyzes will be necessary to better investigate this hypothesis and the functions of these enzymes in cell biology and virulence of these fungi.

\section{ACKNOWLEDGMENTS}

This study was supported by grants from Fundação Carlos Chagas Filho de Amparo à Pesquisa do Estado do Rio de Janeiro (FAPERJ), Conselho Nacional de Desenvolvimento Científico e Tecnológico (CNPq) and Coordenação de Aperfeiçoamento de Pessoal de Nível Superior (CAPES, Finance Code 001).

\section{AUTHOR CONTRIBUTIONS}

XMS conceived and designed the experiments, performed the experiments, analyzed the data, prepared figures and tables, wrote the paper and reviewed drafts of the paper. MHB and ALSS conceive and designed the experiments, contributed reagents/materials/analysis tools, analyzed the data, prepared figures and tables, wrote the paper and reviewed drafts of the paper. All authors critically revised the manuscript and approved the final version.

\section{REFERENCES}

AGARWAL RR, AGARWAL RL, CHEN X, LUA JL AND ANG JY. 2017. Epidemiology of invasive fungal infections at two tertiary care neonatal intensive care units over a 12year period (2000-2011). Glob Pediatr Health 4: 1-8.

BARATA RA, ANDRADE MH, RODRIGUES RD AND CASTRO IM. 2002. Purification and characterization of an extracellular trypsin-like protease of Fusarium oxysporum var. lini. J Biosci Bioeng 94: 304-308.

CARLILE AJ, BINDSCHEDLER LV, BAILEY AM, BOWYER P, CLARKSON JM AND COOPER RM. 2000. Characterization of SNP1, a cell wall-degrading trypsin, produced during infection by Stagonospora nodorum. Mol Plant Microbe Interact 13: 538-550.

CENDEJAS-BUENO E, KOLECKA A, ALASTRUEYIZQUIERDO A, THEELEN B, GROENEWALD M, KOSTRZEWA M, CUENCA-ESTRELLA M, GOMEZLOPEZ A AND BOEKHOUT T. 2012. Reclassification of the Candida haemulonii complex as Candida haemulonii (C. haemulonii group I), C. duobushaemulonii sp. nov. (C. haemulonii group II), and C. haemulonii var. vulnera var. nov.: three multiresistant human pathogenic yeasts. J Clin Microbiol 50: 3641-3651.

CROUZET J, SOTTO A, PICARD E, LACHAUD L AND BOURGEOIS N. 2011. A case of Candida haemulonii osteitis: clinical features, biochemical characteristics, and antifungal resistance profile. Clin Microbiol Infect 17: 1068-1070.

DAVIS DA. 2009. How human pathogenic fungi sense and adapt to $\mathrm{pH}$ : the link to virulence. Curr Opin Microbiol 12: 365-370.

DUBOVENKO AG, DUNAEVSKY YE, BELOZERSKY MA, OPPERT B, LORD JC AND ELPIDINA EN. 2010. Trypsin-like proteins of the fungi as possible markers of pathogenicity. Fungal Biol 114: 151-159. 
FANG SY, WEI KC, CHEN WC, LEE SJ, YANG KC, WU CS AND SUN PL. 2016. Primary deep cutaneous candidiasis caused by Candida duobushaemulonii in a 68 -year-old man: the first case report and literature review. Mycoses 59: 818-821.

HAËN C, NEURATH H AND TELLER DC. 1975. The phylogeny of trypsin-related serine proteases and their zymogens. New methods for the investigation of distant evolutionary relationships. J Mol Biol 92: 225-259.

HAN Z, KAUTTO L AND NEVALAINEN H. 2017. Secretion of proteases by an opportunistic fungal pathogen Scedosporium aurantiacum. PLoS ONE 12: e0169403.

KHAN ZU, AL-SWEIH NA, AHMAD S, AL-KAZEMI N, KHAN S, JOSEPH L AND CHANDY R. 2007. Outbreak of fungemia among neonates caused by Candida haemulonii resistant to amphotericin $\mathrm{B}$, itraconazole, and fluconazole. J Clin Microbiol 45: 2025-2027.

KIM MN ET AL. 2009. Candida haemulonii and closely related species at 5 university hospitals in Korea: identification, antifungal susceptibility, and clinical features. Clin Infect Dis 48: $57-61$.

KOLATTUKUDY PE, LEE JD, ROGERS LM, ZIMMERMAN P, CESELSKI S, FOX B, STEIN B AND COPELAN EA. 1993. Evidence for possible involvement of an elastolytic serine protease in aspergillosis. Infect Immun 61: 23572368.

LI W ET AL. 2015. Distribution of yeast isolates from invasive infections and their in vitro susceptibility to antifungal agents: evidence from 299 cases in a 3-year (2010 to 2012) surveillance study. Mycopathologia 179: 397-405.

LOCKHART SR. 2014. Current epidemiology of Candida infection. Clin Microbiol Newsl 36: 131-136.

LOWRY OH, ROSEBROUGH NJ, FARR AL AND RANDALL RJ. 1951. Protein measurement with the Folin phenol reagent. J Biol Chem 193: 265-275.

MA W, TANG C AND LAI L. 2005. Specificity of trypsin and chymotrypsin: loop-motion-controlled dynamic correlation as a determinant. Biophys J 89: 1183-1193.

MELO AC, DORNELAS-RIBEIRO M, DE SOUZA EP, MACRAE A, FRACALANZZA SE AND VERMELHO AB. 2007. Peptidase profiles from non-albicans Candida spp. isolated from the blood of a patient with chronic myeloid leukemia and another with sickle cell disease. FEMS Yeast Res 7: 1004-1012.

MUHSIN TM, AUBAID AH AND AL-DUBOON AH. 1997. Extracellular enzyme activities of dermatophytes and yeast isolates on solid media. Mycoses 40: 465-469.

MUSZEWSKA A, STEPNIEWSKA-DZIUBINSKA MM, STECZKIEWICZ K, PAWLOWSKA J, DZIEDZIC A AND GINALSKI K. 2017. Fungal lifestyle reflected in serine protease repertoire. Sci Rep 7: 1-12.

PORTELA MB, SOUZA IP, ABREU CM, BERTOLINI M, HOLANDINO C, ALVIANO CS, SANTOS ALS AND
SOARES RM. 2010. Effect of serine-type protease of Candida spp. isolated from linear gingival erythema of HIV-positive children: critical factors in the colonization. J Oral Pathol Med 39: 753-760.

RAMOS LS, BRANQUINHA MH AND SANTOS ALS. 2016. Different classes of hydrolytic enzymes produced by multidrug-resistant yeasts comprising the Candida haemulonii complex. Med Mycol 55: 228-232.

RAMOS LS ET AL. 2015. Candida haemulonii complex: species identification and antifungal susceptibility profiles of clinical isolates from Brazil. J Antimicrob Chemother 70: 111-115.

RAMOS LS, OLIVEIRA SSC, SOUTO XM, BRANQUINHA MH AND SANTOS ALS. 2017. Planktonic growth and biofilm formation profiles in Candida haemulonii species complex. Med Mycol 55: 785-789.

RAO MB, TANKSALE AM, GHATGE MS AND DESHPANDE VV. 1998. Molecular and biotechnological aspects of microbial proteases. Microbiol Mol Biol Rev 62: 597-635.

REDDY PV, LAM CK AND BELANGER FC. 1996. Mutualistic fungal endophytes express a proteinase that is homologous to proteases suspected to be important in fungal pathogenicity. Plant Physiol 111: 1209-1218.

ROBERT V, CARDINALI G AND CASADEVALL A. 2015. Distribution and impact of yeast thermal tolerance permissive for mammalian infection. BMC Biol 13: 18 .

RODERO L, CUENCA-ESTRELLA M, CORDOBA S, CAHN P, DAVEL G, KAUFMAN S, GUELFAND L AND RODRIGUEZ-TUDELA JL. 2002. Transient fungemia caused by an amphotericin B-resistant isolate of Candida haemulonii. J Clin Microbiol 40: 2266-2269.

RUAN SY, KUO YW, HUANG CT, HSIUE HC AND HSUEH PR. 2010. Infections due to Candida haemulonii: species identification, antifungal susceptibility and outcomes. Int J Antimicrob Agents 35: 85-88.

SANTOS ALS. 2011. Protease expression by microorganisms and its relevance to crucial physiological/pathological events. World J Biol Chem 2: 48-58.

SANTOS ALS, CARVALHO IM, SILVA BA, PORTELA MB, ALVIANO CS AND DE ARAUJO SOARES RM. 2006. Secretion of serine peptidase by a clinical strain of Candida albicans: influence of growth conditions and cleavage of human serum proteins and extracellular matrix components. FEMS Immunol Med Microbiol 46: 209-220.

SANTOS ALS AND SOARES RM. 2005. Candida guilliermondii isolated from HIV-infected human secretes a $50 \mathrm{kDa}$ serine proteinase that cleaves a broad spectrum of proteinaceous substrates. FEMS Immunol Med Microbiol 43: 13-20.

SOUTO XM, RAMOS LS, BRANQUINHA MH AND SANTOS ALS. 2019. Identification of cell-associated and secreted serine-type peptidases in multidrug-resistant 
emergent pathogens belonging to the Candida haemulonii complex. Folia Microbiol 64: 245-255.

TSUBOI R, SANADA T, TAKAMORI K AND OGAWA H. 1987. Isolation and properties of extracellular proteinases from Sporothrix schenckii. J Bacteriol 169: 4104-4109.

VAJDA T AND SZABÓ T. 1976. Specificity of trypsin and alpha-chymotrypsin towards neutral substrates. Acta Biochim Biophys Acad Sci Hung 11: 287-294.

VERMELHO AB, MAZOTTO AM, MELO AC, VIEIRA FH, DUARTE TR, MACRAE A, NISHIKAWA MM AND DA SILVA BON EP. 2010. Identification of a Candida parapsilosis strain producing extracellular serine peptidase with keratinolytic activity. Mycopathologia 169: 57-65.

WHALEY SG, BERKOW EL, RYBAK JM, NISHIMOTO AT, BARKER KS AND ROGERS PD. 2016. Azole antifungal resistance in Candida albicans and emerging non-albicans Candida species. Front Microbiol 7: 2173.

ZARNOWSKI R, CONNOLLY PA, WHEAT LJ AND WOODS JP. 2007. Production of extracellular proteolytic activity by Histoplasma capsulatum grown in Histoplasmamacrophage medium is limited to RFLP class 1 isolates. Diagn Microbiol Infect Dis 59: 39-47.

ZHANG Y, LIU X AND WANG M. 2008. Cloning, expression, and characterization of two novel cuticle-degrading serine proteases from the entomopathogenic fungus Cordyceps sinensis. Res Microbiol. 159: 462-469.

ZIMMERMAN M AND ASHE BM. 1977. Substrate specificity of the elastase and the chymotrypsin-like enzyme of the human granulocyte. Biochim Biophys Acta (BBA) - Enzymology 480: 241-245. 